\title{
Design of a Remote Data Monitoring System based on Sensor Network
}

\author{
Niu Ling ${ }^{1}$ and Duan Mei-Xia ${ }^{2}$ \\ ${ }^{1}$ Zhou Kou Normal University, Zhoukou 466001, China; \\ ${ }^{2}$ North China University of Water Resources and Electric Power, Zhenzhou \\ 450011, China \\ Niuling@zknu.edu.cn,Duanmeixia@ncwu.du.cn
}

\begin{abstract}
Aiming at the current situation of remote data monitoring, the study uses the sensor network to realize automation of remote data monitoring. This monitoring system is developed by Internet of Things technology, software technology, network evaluation technology and so on. The system has the characteristics of data real-time processing, visualization, and alarm on abnormal occasion, and has the ability to monitor data remotely and automatically.
\end{abstract}

Keywords: Sensor network; Linux; system assessment

\section{Introduction}

In recent years, with the rapid development of wireless communication, integrated circuit, embedded computing and MEMS (Micro-Electro-Mechanical system) technology. Micro wireless sensor began to appear in the world which has the sense ability, computation and communication capabilities. The sensor has the low cost, low power consumption, multiple functions and so on. The Sensor network is composed of these tiny sensor nodes, so it can easily have real time monitoring, sensing and collecting a variety of environmental or monitoring information, dealing with these data to obtain detailed and accurate information, transmitted the information to the users who need it. Therefore, a sensor network is very important to the information sensing and acquisition, It can provide a large number of detailed and reliable information at any time, any place and any environmental conditions, So it can be widely used in such fields, such as military struggle, national security, environmental monitoring, traffic management, manufacturing, fighting natural calamities anti-terrorism and other fields.

Sensor network is composed of a large number of micro sensor nodes. These low cost sensors are wildly used in the monitoring region; they generally formed a self-organized network system through wireless communication. In the wireless sensor networks, some sensor nodes collect information about the object and also process this information, some sink node sends the information to the observer. We can directly perceive the objective world through a sensor network, which greatly extends the existing network features and the human ability to understand the world. Timely obtaining the information of the sensing area , processing and forwarding the information is one of the key problems in the applications of Wireless Sensor Networks. In general, the sink node receives the data transmitted from sensor nodes, and the sink node is connected with other control devices through wired or wireless mode, the control devices complete the data read, conversion and forwarding work.

Sensor network has become one of the hot issues in a new round development of economic, sciences and technology in the current world. The use and development of it are very meaningful to promote economic development and social progress. In today's 
China, the sensor network has been applied in the fieldsof security, power, transportation, logistics, medical, environmental protection, and the application models are becoming more and more mature. Data monitoring systems based on sensor networks can be widely used in all kinds of the fields of data monitoring. For example, hydrological monitoring, temperature monitoring, environmental monitoring and so on.

China is a vast country, compared with other countries, China is still in the initial stage in data monitoring. The monitoring data are mostly manual samplings, sample analysis is performed by using a handheld portable monitor, or in the laboratory. This way of working is unable to monitor in real time, to sample in high frequency. To reflect the continuous change of parameters dynamically. In addition, some specific monitoring data such as Hydrological monitoring parameters, they are distributed widely, sampled hardly. The sampling time is not fixed. If the testing system consists of the existing manual sampling, wired or wireless network, there are difficulties in construction, maintenance and upgrade, etc.

China also has established its own on-line monitoring system. This system uses the single-chip microcomputer as the host machine, uses the monitoring instrument as the lower position machine, the system monitors the specific data online through the RS232 serial communication. But the data monitored by this kind of system is not precise. With the increase of the monitoring point, the communication cost will be too expensive to bear.

With the low cost of terminal nodes of IOT, the terminal nodes are easily deployed in a different area, which ensures the breadth and precision of data acquisition, All of these can provide basic for monitoring data in large-scale area. The study is to set up a remote data monitoring system which based on Internet of Things technology, software technology, network evaluation technology and so on. In this system, sensors as terminal test nodes, the Internet of things technology as the communication platform, Linux system as the software platform, This kind of construction realized the real-time remote monitoring in the large-scale area.

\section{Overall Design of a Data Monitoring System based on Wireless Sensor Network}

Monitoring system based on Wireless Sensor Network is usually composed of sensors of various parameters, data acquisition equipment, computers, Monitoring software of monitoring information processing. The typical topology structure of Data acquisition is given firstly. According to the application scenarios and the underlying network transmission medium, the sensor network can be divided into wired sensor network and wireless sensor network. Data acquisition technology is more mature in wired sensor networks, which is widely used in industrial, military and other fields. They generally use monitoring instruments from different manufacturers, The communication interfaces of these instruments are various types, communication protocol commonly used with USB, Ethernet, RS-232/485, GPIB etc.

The monitoring software is responsible for the sensor information acquisition, processing, storage and display, which is an important part of the monitoring system. According to the monitoring software scale, the monitoring software can be divided into the single machine monitoring software system and network monitoring software system. The single machine monitoring software system contains all the software subsystem or functional module running on the same computer, data acquisition, calculation processing, storage and user access are concentrated running on this computer. In the software system for network monitoring, through the reasonable division of functions, each subsystem is distributively running on multiple computers, These computers are connected through the network system, each subsystem is cooperating to complete all the functions of the network monitoring software system, 
According to data storage mode, the network monitoring system can be divided into the centralized storage network monitoring system and distributed storage network monitoring system. The main characteristics of centralized storage network monitoring system are to centralizedly store a variety of monitoring information from a variety of distributed environments; this storage method is convenient for data management, maintenance and access. This storage method usually is requires the backbone network to have sufficient bandwidth, centralized storage hardware and software system with high reliability, it is suitable for highly centralized access. Its main disadvantage is when the backbone network implementation or centralized storage work failure, it will affect the whole monitoring system to work normally causing relatively serious losses, Unlike the centralized storage monitoring system, the sensor data information of a distributed monitoring system stored in different computers, These computers are connected through their respective software interface with the centralized management, data scheduling computer. Centralized management and dispatching center is in charge of the management of the whole distributed system, receiving user's data access request, to meet the functional requirements of users access via the data scheduling.

\section{Data Monitoring System Model based on Sensor Network}

In this study, the sensor is used as the terminal test node, the technology of the Internet of things is used to communicate, and Linux system is used as the software platform. Different sensors are used to the different system for different purposes.

\section{1 The Construction of the Hardware Monitoring Platform}

The hardware structure of this data monitoring system mainly includes three parts: the terminal node, the gateway routing node (center gateway, border gateway), remote central monitoring node. Each node performs different functions. The terminal nodes of remote data monitoring system, improve on the performance of power management, network routing algorithm, network communication protocol and the center monitoring software system, So in the course of construction, we should take these factors into account and use the hardware and software which can meet its requirements. The actual hardware topology is shown in Figure 1.

In the remote data monitoring system based on Sensor Networks, you can choose different sensors for detecting various parameters. The terminal nodes can transform the data parameter into data modulated signal through the sensor, then modulates the radio frequency signal and generates a modulated signal, and then the modulated signal is sent to the gateway node through the terminal node of the antenna for data fusion and aggregation.

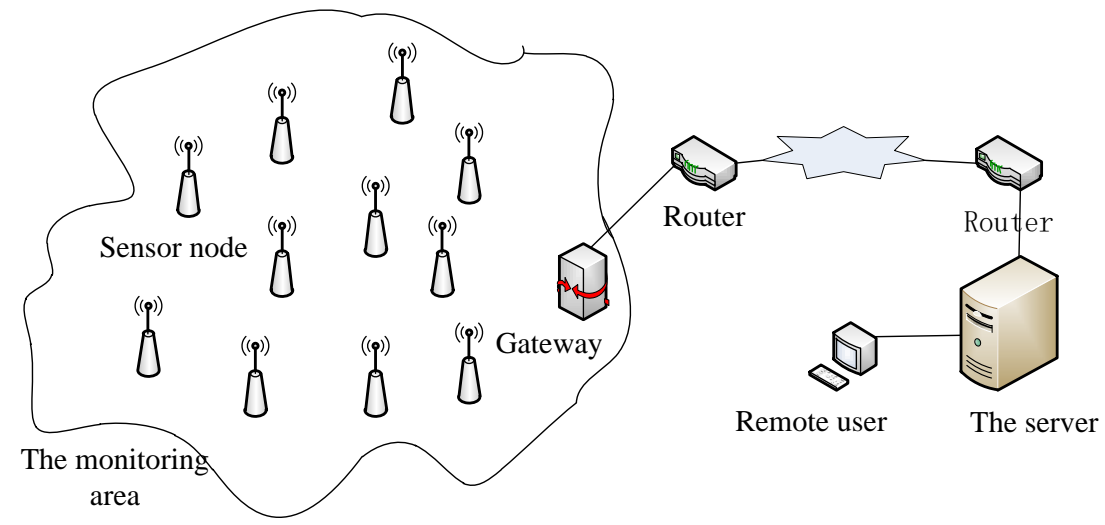

Figure 1. The Hardware Topology 
Each node of monitoring terminal includes data acquisition module (various types of sensors), data processing and control module (microprocessor, memory), communication module (wireless transceiver) and power supply module. In the design it is very necessary to consider these factors (e.g. Node efficiency, node reliability, economic cost and so on), the terminal node can be adjusted according to the need, the work required is only adding terminal nodes of different data parameter test.

The gateway routing node is used to realize the self coordination network and information processing of the entire monitoring sub segment. In the process of establishing the data monitoring network, the key of work test is different due to the different specific environment, so the different segment requires a separate setting. First of all, each gateway node should initialize its segment to avoid mutual interference of terminal nodes, and other signal interference. The gateway node uses different physical addresses to each terminal node in the network to distinguish different node, the gateway node periodically sends a query command after the whole network application. When discovering a new network node the system will automatically add it to the network node list, and send the new routing table.

In addition to self-organized characteristics, the gateway node is responsible for information analysis and processing firstly, and stores the processed data to the embedded database for the query. The gateway node is usually limited in number, generally the power requirements are not strict, can use a variety of means of communication with other nodes of the network (such as Internet, satellite or mobile communication network). In the monitoring data system of the internet of things, star type topology design is adopted.

The remote central monitoring node is the management center of the whole system, collecting and processing the data parameters of each region, and put forward different reasonable suggestions according to the results of the analysis, complete data storage and processing, data visualization, the management of the Internet of things. The hardware is mainly the large-scale disk array and high performance workstation server.

In the whole data monitoring system, after the completion of Hardware deployment and software parameter settings, you can intuitively monitor the parameters of the terminal sensor node region. The specific process is as follows:

(1) The remote monitoring center sends out the control command, through the gateway node, activate the terminal sensor node to detective parameters.

(2) When the terminal node processor receiving the instruction, the command is decoded by the main processor. If the node address and a control instruction address are consistent, start sensor to collect data, and transfer the final collected data to the node processor. After the node main processor capturing the measuring data, it analyses and fuses related data and packs the data into the data frame which meets the 6LoWPAN protocol standard, then adds a packet header, node number and other information. Then the packed data were sent to the radio frequency module for transmitting, at the same time other node's routing also can be transmitted in the node.

(3) Center node converges each terminal node parameter, and sends out the corresponding control instruction.

\section{2 Software System Integration and Design}

For the convenience of the different module interface, the overall software design shall adopt consistent, modular design. Because the Linux system is matured and stable, open source code, and particular has its unique advantages in the aspect of network communication. So all the node platforms for Development and application use the Linux operating system.

In the data monitoring system, the management function is more complex, tasks and monitoring parameters are varied. So must set aside software interfaces for 
other parameter testing software in the future. The terminal nodes due to its energy saving aspects, can use the minimum embedded Linux operating system after cutting, the gateway node uses the common embedded Linux operating system, while the center node using the complete Linux system. This software platform architecture ensures the software, consistency of the whole system, in order to facilitate the future security and maintenance.

\section{2.1 Software Design of the Terminal Node}

Terminal node hardware platform mainly includes the core controller, I/O interface, memory module and RF transceiver module. Its hardware structure determines the terminal node with minimum embedded Linux operating system after cutting, must use the revised protocol stack to realize message fragmentation and reassembly, header compression, address auto configuration, multicast and security. Protocol stacks data frame format conforms to IEEE802.15.4, the data parameter testing protocol frame format is shown in Figure 2. The MAC load part includes control information of the upper layer protocol frame and data parameters, sensor node number and other information.

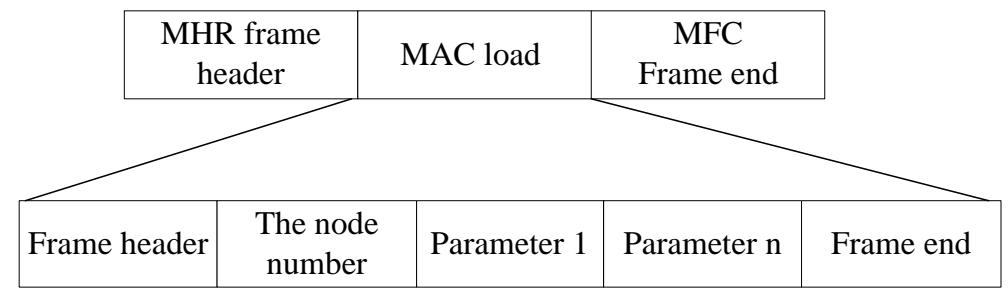

\section{Figure 2. The Testing Protocol Frame Format}

As the terminal nodes, the software design of energy saving is an important consideration. The terminal nodes use the working mode based on the threshold: when the monitoring data size is below the threshold, not to send; when the monitoring data size is between a reporting threshold and alarm threshold, circularly report the real-time data in a long time; when the monitoring data size exceeds the alarm threshold; circularly report the real-time data in a shorter time. The working mode not only ensures reliable access to critical real-time data, but also reduces the energy consumption by frequently sending useless data.

\section{2.2 Software Design of Monitoring Center}

The monitoring center application software is as friendly as possible for the user to operate, displaying the danger or abnormal condition clearly and alarming clearly, summarizing and analyzing the data parameters, communicating with the bottom.

Based on the requirements of design target, the monitoring center software platform uses $\mathrm{B} / \mathrm{S}$ framework. Monitoring center application realizes data monitoring, network management via the core platform, and start the terminal data monitoring nodes to sample the regional data. When the monitoring center collecting sample data sent by routing nodes, the data can be stored in the backstage database, at the same time, the monitoring center provides a user interface to analyze and process the data parameters, using graphics display, finally give a visual or audible alarm according to the results of the analysis. The system application program mainly consists of 4 functional modules: document processing module, system configuration module, the analysis and processing module and alarm module.

The software system platform of monitoring center is a Linux system, the database is an ORACLE large relationship database. 
The document processing module can realize the data storage, retrieval and printing function, can also save the application work environment parameters, in order to comprehensively cope with later data.

The system configuration module can realize the hardware and software configuration of the entire data monitoring system, including the detection of hardware when the application starts, the software and hardware initialization environment after the hardware detection.

The analysis and processing module is the core module of the monitoring center, mainly includes the analysis of monitoring data, classification, comparison, model building, data normalization, data curve plotting and display. Generally include the following 4 aspects:

(1) With geographic information systems, display all terminal node positions and real time data on the map, which can realize fast positioning geographic coordinates of data terminal node for the monitor

(2) Time is the x-axis, all terminal node history or the real-time data are on the y-axis. According to the curve graph, the monitor can analyze the change of data parameters in a period of time. Then combining with other monitoring information, analysis, improve the early warning mechanism;

(3) Based on the node ID display, according to the size of logo, display all the real-time data and the state of the node, so the monitor can extract monitoring data and observe the state of the node, maintaining the whole network system in a timely and effective manner.

(4) The construction of the data parameter model, the parameter prediction model and the expert system, they provide a scientific basis for the leadership decision-making. The alarm function module can realize to alarm the abnormal conditions on the data parameters. Mainly achieve fast positioning of abnormal nodes and alarm, according to a set of exception handling strategy. Alarm modes include in a graphical interface, fast flashing red alarm information, alarm prompting sound emitted by the speaker.

\subsection{System Evaluation:}

In the data monitoring system, a large number of data sampling terminal nodes are deployed in different areas. Each terminal node constitutes a network in wireless way to communicate. The wireless signal transmission has the multipath effect caused by reflection and diffraction, in addition the nodes are in complex environments, thus causes the network communication quality to be affected badly. Finally, these factors lead to the communication instable between nodes in the network. This situation may lead to the whole data monitoring system cannot work in stability. The monitoring data collected cannot be timely and accurately transmitted to the monitoring center for analyzing and decision making.

After the success of the system set up, using for reference to domestic and foreign project evaluation method based on the Internet of things system (such as based on the communication link characteristics evaluation, based on the test parameters evaluation, combined with multi factor, comprehensive evaluation), we applied these evaluations to this system, including the RF parameters, testing and evaluation of a terminal node, the accuracy and reliability evaluation of the sampling data parameters, and the evaluation of the network communication quality and the network life cycle. These specific evaluation methods will make the system operate at high efficiency and reliability. 


\section{Conclusion}

Data monitoring systems based on the Internet of things technology is flexible and easy in nest building, physical limitations are loose, can easily realize the data parameter test network. We can also easily monitor online and real-time data of the test area by adding a terminal point on the network without changing the overall structure of the system. The construction of the Internet of things can be made in some inaccessible region of remote monitoring data, which is very meaningful for disaster monitoring.

\section{Acknowledgement}

This Research is supported by the Natural Science of China Foundation (61103143).

\section{References}

[1] D. Chakrabarti, S. Maitra and B. Roy, "A Key Pre-distribution Scheme for Wireless Sensor Networks: Merging Blocks in Combinatorial Design”, Journal of Information Security, vol. 5, no. 2, (2006), pp. 105-114.

[2] V. Kawadia and P. R. Kumar, "Principles and Protocols for Power control in Ad Hoc networks", IEEE Journal on Selected Areas in Communications, vol. 23, no. 1, (2005), pp. 76-88.

[3] W. R. Heinzelman, A. Chandrakasan and H. Balakrislman, "Energy-efficient communication Protocol for wireless micro sensor networks", System Sciences, Proceedings of the $33^{\text {rd }}$ Annual Hawaii International Conference on Maui, USA, IEEE, (2000); Los Alamitos, CA, USA.

[4] O. Y. and S. F. Heed, "A Hybrid, Energy-Efficient, Distributed Clustering Approach for Ad Hoc Sensor Networks", IEEE Transactions on Mobile Computing, vol. 3, no. 4, (2004), pp. 366-379.

[5] K. S. and W. M. and J. E, "Scalable self-assembly for Ad hoc wireless sensor networks", IEEE Transactions on Mobile Computing, vol. 3, no. 4, (2004), pp. 317-331.

[6] M. C. and L. Z., "An implicit prioritized access protocol for wireless sensor networks", Proc. of IEEE Real-Time System Symposium, (2002); Austin,Texas.

[7] W. Y., "An energy-efficient MAC Protocol for wireless sensor networks", Proc of the 21st International Annual Joint Conference (Infoeom), IEEE Computer and Communications Societies, (2002); New York.

[8] D. Braginsky and D. Estrin, "Rumor routing algorithm for sensor networks", Atlanta, GA, United States, Association for Computing Machinery, (2002), pp. 22-31.

[9] S. P., S. P., B. P. and G. S., "Au unifying framework for tunable topology control in sensor networks", University of Southern California: Technical Report, (2005).

[10] J. B. W., M. G. D. and A. K. Castner, "Analyzing interaction between distributed denial of service attacks and mitigation technologies", DARPA Information Survivability Conference and Exposition, (2003); Washington, DC, USA.

[11] M. Y. and K. G., "Location-Aware combinatorial key management scheme for clustered sensor networks", IEEE Trans on parallel and Distribution System, vol. 17, no. 8, (2006), pp. 865-882.

[12] H. Zhang and A. Arora, "GS3:Scalable self-cofiguration and self-healing in wireless sensor Networks", Computer Networks, vol. 43, no. 4, (2003), pp. 459-480.

[13] X. Wang and T. Berger, "Self-organizing redundancy-cellular architecture for wireless sensor networks", IEEE Wireless Communication and Networking Conference, WCNC, Institute of Electrical and Electronics Engineers Inc., (2005); New York, United States.

[14] X. Wang and T. Berger, "Topology control resources allocation and routing in wireless sensor networks, Proc of the 12th IEEE MASCOTS, IEEE Computer Society, Los Alamitos, (2004); United States.

[15] L. Blain and Y. Deswarte, "An Intrusion-tolerant Security Server for An Open Distributed System, Proceedings of the European Symposium in Computer Security, (1990).

[16] C. F. Grecas, S. I. Maniatis and I. S. Venieris, "Introduction of the Asymmetric Cryptography in GSM, GPRS, UMTS, Its Public Key Infrastructure Integration, (2003).

[17] M. Blaze, J. Feigenbaum and J. Lacy, "Decentralized Trust Management", Proceeding of the 17th Workshop on Security and Privacy, IEEE Computer Society Press, (1996); Oakland, USA.

[18] S. Ganeriwal and M. Srivastava, "Reputation--based Framework for High Integrity Sensor Networks, Proceedings of the 2nd ACM work shop on security of Ad Hoc and Sensor Networks, (2004).

[19] J. Hur, Y. Lee and S. M. Hong, "Trust Management for Resilient Wireless Sensor Networks", Proceedings of the ICISC, (2006); Berlin, Germany. 


\section{Authors}

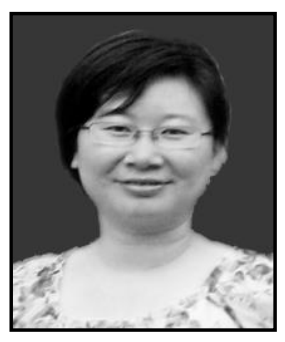

Niu Ling, she received the B.Eng degree in Computer science from Henan normal university and M.Eng degree in Computer science from Chengdu University of Technology. She is currently researching on computer application technology.

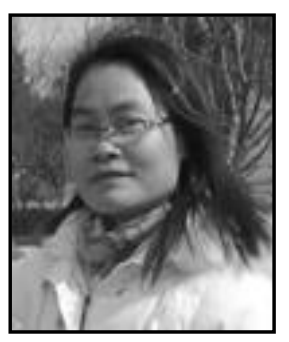

Duan Mei Xia, she received the B.Eng degree electronic technology in from Henan normal university and M.Eng degree in Computer science from Chengdu University of Technology. She is currently researching on embed system and measure . 